\title{
Pain-elicited aggression in the squirrel monkey: An implicit avoidance contingency
}

\author{
PHILIP J. DUNHAM and ADAM CARR \\ Dalhousie University, Halifax, Nova Scotia, Canada
}

\begin{abstract}
Squirrel monkeys were given either forward pairings of a bite-tube CS and shock US or backward pairings of these stimuli. Backward pairings produced stronger control of biting by the bite tube alone than did forward pairings. In a second experiment, subjects received backward pairings of US and CS with either a fixed ITI or a random ITI. Conditioned biting was obtained only when trials were presented with a fixed ITI. The magnitude of unconditioned biting was also significantly greater with the fixed ITI. It was argued that these results demonstrate that conditioning in this situation depends upon the degree to which biting predicts a relatively long shock-free period. When trials occur randomly in time, biting predicts no definite shock-free period; hence, it is not learned.
\end{abstract}

In the course of discussing methods for analyzing the effects of punishment procedures upon behavior, Dunham (1971) has suggested that certain procedures used to present aversive stimuli to an animal can establish an "implicit" avoidance contingency for the response which is typically elicited by the aversive stimulus. Consider, for example, an hypothetical organism which exhibits only two behaviors, $A$ and $B$. If a periodic electric shock is introduced into this organism's world, a third response, E, elicited by the shock, is generally added to the response repertoire. The elicited response, because it is sequentially dependent upon the occurrence of the shock, initially enjoys the unique property of predicting the absence of shock for a longer period of time than any other behavior in the repertoire (either response A or B). This "implicit" avoidance contingency would, of course, be eliminated if the intershock interval were short enough to permit the elicited behavior to be punished by the onset of subsequent shocks.

There are at least two phenomena in the contemporary literature in which this "implicit" avoidance contingency could play an important role. The first has emerged from the context of research concerned with the effects of extended exposure to periodic shock upon the aggressive behavior of the squirrel monkey; the second emerged from research which attempts to demonstrate that aggressive behavior can be classically conditioned to previously neutral stimuli.

With reference to the first of the above cases, O'Kelly and Steckle (1939) were apparently the first investigators to report that brief, painful stimulation will elicit aggressive behavior between pairs of rats exposed to such stimulation. The generality of this phenomenon, typically called pain-elicited aggression, has subsequently been demonstrated in a variety of experimental

This research was supported by Project Grant APA-194 to Philip J. Dunham from the National Research Council of Canada. The authors would like to thank Patrick Kelly, Pat Thomas, and Robyn Pascoe for their assistance on all phases of this project. Requests for reprints may be sent to either author. Department of Psychology, Dalhousie University, Halifax, Nova Scotia, Canada. procedures using a wide variety of species (Hutchinson, 1973).

One of the frequently used preparations used for studying the painelicited phenomenon involved placing a squirrel monkey in a restraining chair and delivering periodic shocks to the animal's tail. Under such conditions, the monkey will reliably attack (bite) a length of rubber tubing located in front of its face (Hutchinson, Azrin, \& Hake, 1966). In the course of studying the effects of extended exposure to periodic shock upon the attack behavior of the squirrel monkey, Hutchinson, Renfrew, and Young, (1971) and Hutchinson and Emley (1972) have consistently observed two interesting changes in the biting behavior. First, when the shock is presented at relatively long intershock intervals and higher intensities, there is an increase or facilitation of elicited biting behavior over trials. Indeed, in the latter conditions the monkey will continue its biting well into the intershock interval, stopping only during the short interval immediately prior to the onset of the next scheduled shock. Once this within-session facilitation of biting has been established, it is possible to discontinue the shock and observe the increased levels of biting persist for several subsequent shock-free sessions.

Hutchinson et al. (1971) suggested that the decrement in elicited biting observed with the short intershock intervals and mild shock intensity can be interpreted in terms of a habituation process whereby the shock loses its aversive properties with repeated presentations. The facilitation of biting observed with longer intershock intervals and higher shock intensities was interpreted in terms of a classical conditioning process whereby apparatus cues and other environmental stimuli are assumed to acquire attack-eliciting properties via pairings with the periodic shock.

We would suggest that the "implicit" avoidance contingency described earlier offers an alternative explanation for the facilitation of biting observed by Hutchinson et al. (1971). When relatively intense shocks are presented using long intershock intervals, the elicited biting response predicts the absence of shock 
for a longer period of time than any other behavior which might be occurring in this situation. Similarly, one would not expect the contingency to be effective with short intershock intervals if such intervals permitted punishment of the elicited biting behavior early in training. It is not possible to determine from the procedures used by Hutchinson et al. (1971) the extent to which the classical conditioning process they have described and/or the "implicit" avoidance contingency outlined earlier were operating to produce the facilitation of pain-elicited biting.

The second, and related, case in which the "implicit" avoidance contingency can be implicated concerns the attempts by a number of investigators to classically condition pain elicited biting behavior. Generally speaking, the literature indicates that pain-elicited aggression has been curiously resistant to the classical conditioning operation.

Vernon and Ulrich (1966) attempted to condition pain-elicited fighting between pairs of rats using a one-second tone as a CS and various levels of shock intensity as the UCS. After some 2,000 pairings, fighting was observed on only $50 \%$ of the test trials in which the CS was presented alone. Creer, Hitzing, and Schaeffer (1966) used a very similar procedure and obtained only minimal conditioning after several thousand trials.

More recently, Lyon and Ozolins (1970) varied both CS duration and the intertrial interval in an attempt to classically condition pain-elicited fighting behavior in rats. Using a discrimination procedure with either a tone or a clicking noise as conditioned stimuli, they observed no evidence of conditioned fighting behavior if the intershock interval was less than $64 \mathrm{sec}$. With intervals of $64 \mathrm{sec}$ between shock presentations, the CS+ in their procedure did elicit some fighting behavior. With extensive training at this interval the conditioning performance reached an asymptote of 50\% during daily sessions. It is interesting to note at this point that the intershock intervals employed by Vernon and Ulrich and by Creer et al. were $10 \mathrm{sec}$ and $3 \mathrm{sec}$, respectively.

Perhaps the strongest evidence for the conditioning of pain-elicited aggression has been reported by Hutchin. son, Renfrew, and Young (1971, Experiment III) as a part of the work mentioned earlier. These authors paired either a light or a tone with intense electric shock delivered to the tails of squirrel monkeys restrained in a chair and provided with a bite tube. With an intershock interval of $4 \mathrm{~min}$, the tone CS elicited vigorous biting within 25 trials. There was little evidence for conditioning using the light as a CS. This difference between the tone and light CS might be explained if one assumes that the monkeys were directing their attacks at the light CS rather than at the tube (cf. Rachlin, 1969; Moore, 1973 , p. 172). It should be noted that even in monkeys showing vigorous asymptotic biting in response to the tone CS, there was some intertrial biting occurring in this experiment.
To summarize briefly, the evidence suggesting that pain-elicited aggression can be classically conditioned is sparse and relatively weak. The experiments just described differ in a number of important details, some of which can quite obviously be blamed for the failure to obtain strong conditioning (e.g., short CS duration, latent inhibition trials, etc.). The implicit avoidance contingency described by Dunham suggests, however, that the intershock interval used in the conditioning procedure may also be an important variable. It is only with long intershock intervals that the elicited biting behavior will reliably predict a safe period and escape additional punishment. The data discussed thus far support this contention. The most effective conditioning procedures have been those with the longest intershock intervals, although none suggest that conditioning painelicited aggression is particularly easy.

The experiments to be reported in the present paper represent our initial attempts to investigate the role which the implicit avoidance contingency discussed earlier might play in the modification of pain-elicited aggression observed in signaled and unsignaled shock procedures. We would suggest that this contingency may play an important part in the facilitation of biting observed with periodic unsignaled shock, and in the success or failure of attempts to classically condition pain-elicited aggression.

\section{EXPERIMENT I}

One method for determining the extent to which the implicit avoidance contingency contributes to facilitation and conditioning of pain-elicited biting is to use a backward conditioning procedure in which (a) a CS predicts a safe interval rather than the onset of shock, and (b) the elicited biting behavior is restricted to a brief period following the shock to prevent the accidental punishment of biting. The backward pairing of CS and shock rules out the possibility of biting being classically conditioned to the CS (unless one wants to consider the possibility of strong, excitatory, backward conditioning), but leaves the implicit avoidance contingency described by Dunham intact. If as a result of such backward pairings we observe an increase in unconditioned biting, and the CS elicits biting when presented alone, we have some strong evidence for the operation of the avoidance contingency (or backward conditioning), independent of the classical conditioning mechanism discussed by Hutchinson et al. (1971). The procedure is considered particularly stringent in the sense that it actually asks the animal to exhibit what can be considered a case of backward excitatory conditioning, a phenomenon not generally observed (cf. Heth \& Rescorla, 1973). Indeed, according to experiments reported by Moscovitch and LoLordo (1968) and Siegel and Domjan (1971), the backward pairing procedure should produce a CS which inhibits the motivational state aroused by anticipation of shock. 


\section{Method}

Subjects. Eighteen adult male squirrel monkeys (Saimiri sciureus) obtained from Connaught Laboratories, Willowdale, Ontario, served as subjects. They were housed in cages $36 \mathrm{~cm} \mathrm{x}$ $47 \mathrm{~cm} \times 36 \mathrm{~cm}$ which had stainless steel walls, grid floor, and plastic ceiling to permit observation via overhead closed-circuit television. Food and water were freely available in these cages except during experimental sessions.

Apparatus. The housing cages served as experimental chambers when moved to an adjacent room. The conditioned stimulus (CS) consisted of the presentation of a semicircular configuration of $2 \mathrm{~cm}$ diam surgical tubing through a slot $(15 \mathrm{~cm} \mathrm{x}$ $3.5 \mathrm{~cm}$ ) in the front wall of the chamber $21 \mathrm{~cm}$ above the grid floor. The CS was accompanied by the noise of a motor-cam mechanism used to insert and retract the tube. The noise continued for the duration of the CS which was nominally $20 \mathrm{sec}$, resulting in the stationary presence of the tube for $18 \mathrm{sec}$ during each CS period. The unconditioned stimulus (US) was scrambled electric shock delivered via wall and floor grids from a Grason-Stadler E1064 shock generator set at $5 \mathrm{~mA}$ for $.5 \mathrm{sec}$. Any bites on the bite tube closed an air-pressure switch which activated appropriate recording equipment in the control room. This procedure deviates from the usual method of studying pain-elicited biting attacks in several respects. Our monkeys are not restrained in a chair, the bite tube can be inserted or removed, and the shock is delivered via grids rather than electrodes on the tail. Observations made with the closed circuit monitor indicated that shocks were delivered reliably via the electrified grid floor and/or walls of the chamber. Direct observation also permitted us to eliminate occasional switch closures produced when the monkey grabbed or bumped the bite-tube.

Procedure. The 18 monkeys were randomly assigned to 3 equal-sized groups. The primary group of interest following from the preceding rationale was the backward pairing group (BP) in which shock was followed immediately by the insertion of the tube for $20 \mathrm{sec}$. A forward pairing group (FP) was also included to determine the extent to which we could classically condition the biting attack with this preparation. In this group, the tube was inserted $20 \mathrm{sec}$ prior to the onset of shock and retraction was initiated with shock offset. Finally, a random control ( $R$ ) group was run as a control for nonassociative effects (Rescorla, 1967). These animals received CS and US presentations according to identical, independently programmed random time, 240-sec schedules. In groups BP and FP the intershock interval was $240 \mathrm{sec}$, and the sessions were terminated when a subject had received 10 trials in Groups BP and FP or $10 \mathrm{CS}$ and 10 US presentations in Group R.

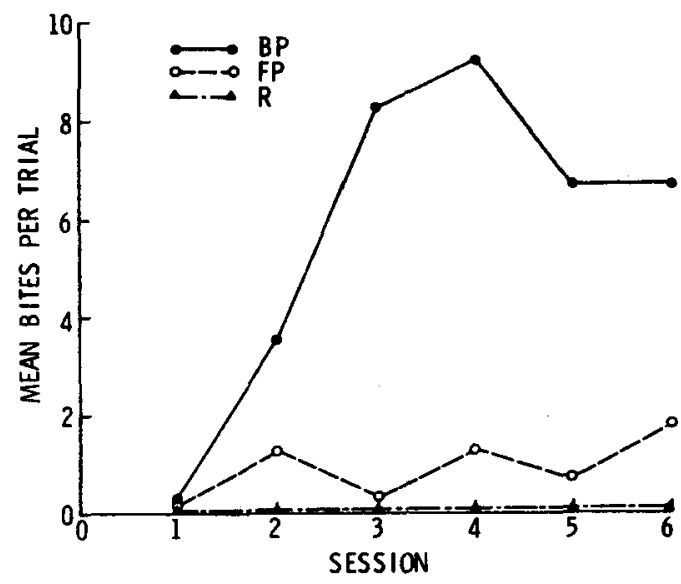

Figure 1. Average bites per Trial 5 (no-shock test trial) across Sessions 1-6.

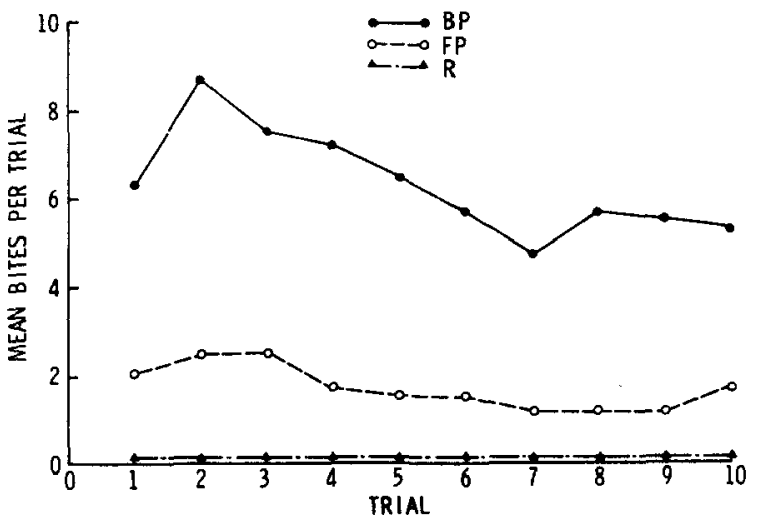

Figure 2. Average bites per trial during Session 7. Trial 1 was a shock trial, followed by no-shock Trials 2-10.

During the first six daily sessions, subjects in Groups BP and FP received nine conditioning trials and one shock-free test trial per session. The test trial was always the fifth daily trial. On the seventh day, one conditioning trial was presented, after which the remaining nine trials were presentations of the CS alone. Subjects in Group $\mathrm{R}$ received $10 \mathrm{CS}$ alone presentations on Day 7.

Throughout the experiment a naive observer continuously recorded whether the subject was in the forward half of the chamber (near bite tube area) or in the back half of the chamber. Pilot work had suggested that there might be systematic differences on this measure between forward and backward conditioning groups.

\section{Results and Discussion}

Analyses of variance were carried out using Rodger's method (Rodger, 1967a, b). With this method unlimited post-hoc data-snooping is possible, at known error rates for Type I and Type Il errors, provided a decision set of $v_{1}$ orthogonal null contrasts is asserted in which $\mathrm{r}=\mathrm{F} / \mathrm{F}[\mathrm{E} a]: v_{1}, v_{2}$ contrasts are rejected and $v_{1}-\mathrm{r}$ asserted. For the following analyses, Type I error rate was set at $\mathrm{Ea}=.05$, while $\mathrm{E} \beta$, which is the expected proportion of null rejections given a noncentral parameter of specified size, was set at .70. Rodger's (privately circulated) tables of $\mathrm{F}[\mathrm{Ea}]: v_{1}, v_{2}$ were used to determine the significance of statistical tests.

Figure 1 shows the average bites per trial during the daily test trial over the six training sessions. No biting was ever observed in Group $R$ during the test trials. In fact, we observed a total of only 11 bites from all subjects in Group $R$ during the seven days of exposure to the random schedule. In Groups FP and BP, conditioned biting had emerged by the second training session, and backward pairings of the US and CS produced a greater magnitude of conditioned biting than did forward pairings. An analysis of variance revealed significant differences among the groups in terms of the average number of bites made on Trial 5 (the test trial in Sessions 1 through 6$),(F=4.50, d f=2 / 15$, $p<.05)$. A similar effect was obtained during the extinction trials (Session 7). Group BP made significantly more bites during extinction than Group FP $[F(2 / 15)$ 


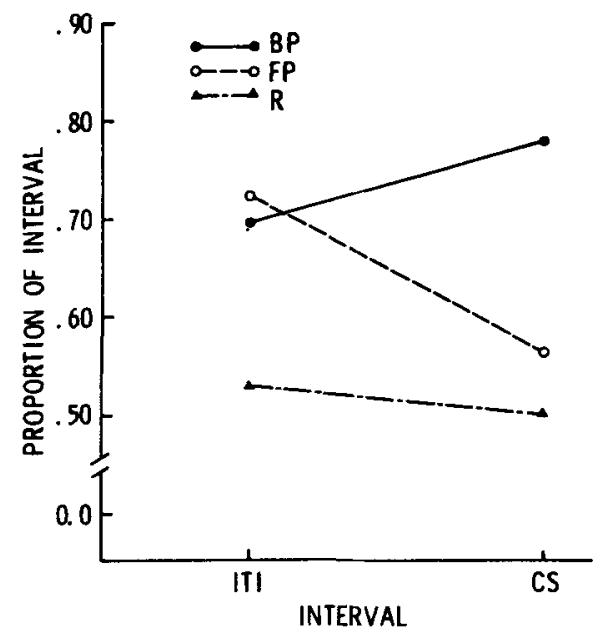

Figure 3. Mean proportions of ITI and CS intervals spent in the front half of the chamber. All trials over Sessions 1-7 are included.

$=3.77, \mathrm{p}<.05]$. Figure 2 presents the average number of bites per trial for each group during the extinction session.

Not all subjects in either Group FP or Group BP showed conditioned biting. In Group BP, the data from four subjects accounted for more than $99 \%$ of all test trial bites, while in Group FP a single subject accounted for all test trial bites. This distribution of biting indicates that conditioned biting may be difficult to obtain in the forward pairing condition, but when obtained it may be as strong as the biting observed in the backward pairing condition.

Figure 3 is a plot of the average proportion of time during ITI and CS periods spent in the front half of the chamber (near the CS area). Monkeys in the BP condition spent significantly more time in the forward half of the chamber during both the CS and ITI periods than the random group, which spent approximately $50 \%$ of each period in the forward half of the chamber. Group FP spent as much time as Group BP in the forward half of the chamber during the ITI when the tube was absent, but tended to go to the back half of the chamber more frequently than BP subjects during the CS. Both BP and FP subjects spent more time in the front half of the chamber during ITI and CS than R subjects.

The data presented lead to the apparently paradoxical conclusion that the backward pairing procedure used in this experiment produces significantly better conditioned biting behavior than the forward pairing procedure.

Consider first the relatively rapid conditioning obtained in Group BP. We would suggest that these results argue strongly for the operation of the "implicit" avoidance contingency described by Dunham (1971). The backward pairing procedure rules out the possibility of strong excitatory classical conditioning, and the random control condition argues against any nonassociative, sensitization interpretation. The elicited biting behavior does, however, predict a longer interval of safety than any other response the monkey might make, and is associated with the biting tube.

Our failure to find strong evidence for forward conditioning of pain-elicited aggressive behavior is compatible with previous evidence indicating some difficulty in obtaining such conditioning. It is, however, somewhat puzzling in that we used the long intertrial intervals used by Hutchinson et al. (1971), who observed some evidence for conditioning. There are, however, at least three factors which might make it difficult to classically condition pain-elicited aggressive behavior in this and other experimental situations. First, and most likely, it is generally assumed that forward pairing procedures like that used in the present study will produce fear conditioning (Rescorla \& Solomon, 1967). It seems reasonable to suggest that many of the responses associated with fear are incompatible with approaching and biting the tube which signals shock. Our measure of the monkeys' cage position during the ITI and the CS can be viewed as support for this interpretation. As indicated in Figure 3, the FP monkeys spent less time in front of the chamber when the CS was present (tube inserted) than when the tube was absent and the slot was empty.

A second possible problem in the forward pairing procedure is that the monkey can learn some type of preparatory response which is instrumental in reducing shock intensity and/or duration; such a preparatory response might be incompatible with biting attacks. The animals in Group FP were observed to make a number of responses to CS onset, and these responses appeared to be under the control of the CS as training progressed (e.g., somersaulting, jumping around, pacing the perimeter of the chamber, etc.). Although the shock was always observed to elicit strong vocalizations, it is impossible to determine from the present data whether these behaviors might have managed to reduce the intensity and/or duration of shock.

Finally, a third factor whcih might contribute to the poor performance in Group FP is unique to the present procedure. The forward pairing procedure used in this first experiment permitted approximately $2 \mathrm{sec}$ of elicited biting as the tube retracted immediately after shock offset. It is possible, though unlikely, that a longer duration of unconditioned biting is required for conditioning to occur.

In summary, the results of the first experiment suggested that a backward pairing procedure which maximizes the operation of the "implicit" avoidance contingency described by Dunham is more effective for conditioning pain-elicited aggression than a forward pairing procedure which presumably maximizes the conditions for classical excitatory conditioning of such behavior. 


\section{EXPERIMENT II}

Although further research will be necessary to determine which of several factors might be responsible for the failure to obtain evidence for forward conditioning of pain-elicited aggression, we decided to concentrate our efforts in this second experiment upon further examination of the avoidance interpretation suggested to account for the conditioning observed in the backward pairing groups.

Since there is some evidence for very weak excitatory conditioning using backward pairing procedures (see Heth \& Rescorla, 1973), it is possible to argue that the conditioned biting we observed with the backward pairing procedure in the first experiment represents a rare case of relatively strong, backward excitatory conditioning. Alternatively, one might suggest that the development of biting observed in the backward pairing procedure represents an example of instrumental escape conditioning whereby the response most closely associated with shock offset develops and is maintained by an escape contingency.

Both of these interpretations can be offered as alternatives to the "implicit" avoidance contingency analysis discussed earlier, and the second experiment was designed to pit these alternative interpretations against the "implicit" avoidance hypothesis. Basically, the experiment involved two groups of monkeys: a backward pairing group with a fixed intertrial interval of 4 min (replicating Group BP, Experiment I), and a backward pairing group with a random intertrial interval with an average of $4 \mathrm{~min}$. The use of the random intertrial interval eliminates the "implicit" avoidance contingency (i.e., elicited biting no longer predicts a safe interval), but maintains the potential for backward excitatory conditioning or escape conditioning. Hence, the "implicit" avoidance hypothesis predicts conditioned biting only in the fixed intertrial interval condition, whereas the alternative interpretations predict conditioned biting in both conditions.

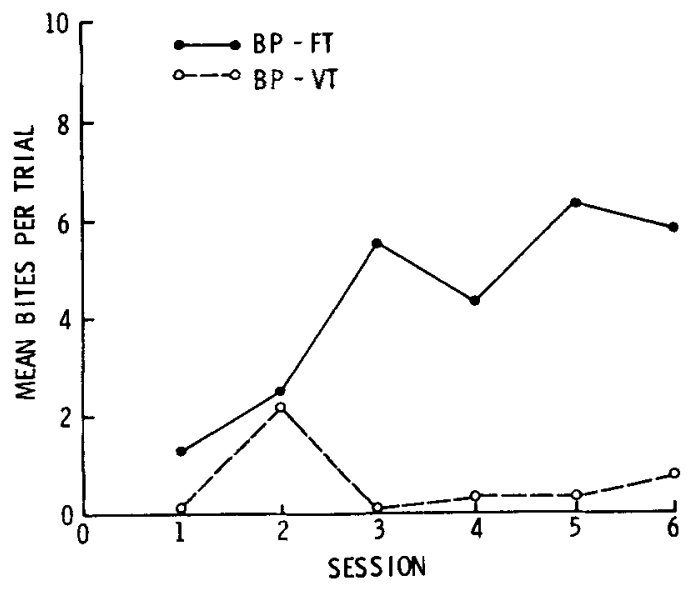

Figure 4. Average bites per Trial 5 (no-shock test trial) across Sessions 1-6.

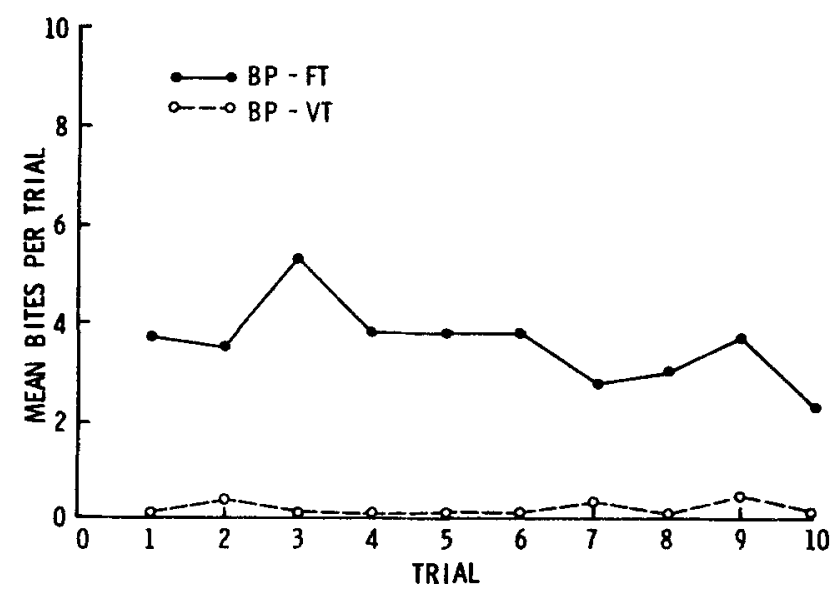

Figure 5. Average bites per trial during Session 7. Trial 1 was a shock trial, followed by no-shock Trials 2-10.

\section{Method}

Subjects and Apparatus. Twelve additional squirrel monkeys were obtained from Connaught Laboratories for this experiment. All details concerning the subjects and apparatus were the same as in Experiment $I$.

Procedure. Subjects were divided into two equal-sized groups. Group BP-FT received backward pairings of the shock US and the bite-tube CS on a fixed time (FT) schedule. Group BP-VT received backward pairings of US and CS on a variable time (VT) 4-min schedule. The variable time schedule programmed the US to occur randomly in time with an average interval of $240 \mathrm{sec}$ and a fixed probability of $1 / 240$ that the US would occur during any given second. As such, the schedule does not program any "safe" interval between trials, since, theoretically, a shock can occur in the first second immediately after a trial.

As in the previous experiments, subjects received 9 conditioning trials and one shock-free test trial per day for 6 days. On the 7 th day, all subjects received 9 consecutive trials on which the CS was presented alone after the first conditioning trial.

\section{Results and discussion}

Figures 4 and 5 show the average number of bites per test trial for Trial 5, Days 1-6 and Trials 1-10, Day 7. Conditioned biting developed during the first conditioning session in Group BP-FT and reached an asymptote of 5-6 bites per trial in the 3rd session. During the test session, Group BP-FT made an average of 3-4 bites per test trial. Very little conditioned biting was observed in Group BP.VT, as can be seen in the figures. Group BP-FT made significantly more bites per test trial than did Group BP.VT over Days 1-6 [F(1/10) $=5.74, p<.05]$ and on Day 7 (Trials $2.10, F=5.20$, $\mathrm{df}=1 / 10, \mathrm{p}<.05)$.

It might be noted also that the implicit avoidance interpretation predicts that the amount of unconditioned biting will increase over sessions, only in the fixed intertrial interval condition. As predicted, the magnitude of unconditioned biting was also significantly less in Group BP.VT than in Group BP-FT $(\mathrm{F}=5.87, \mathrm{df}=1 / 10$, $\mathrm{p}<.05$ ). Figure 6 displays the average postshock bites per trial for Groups BP-FT and BP-VT per Sessions 1-6. Subjects in Group BP-FT made over 5 times as many postshock bites, on the average, as did those in Group BP-VT. 


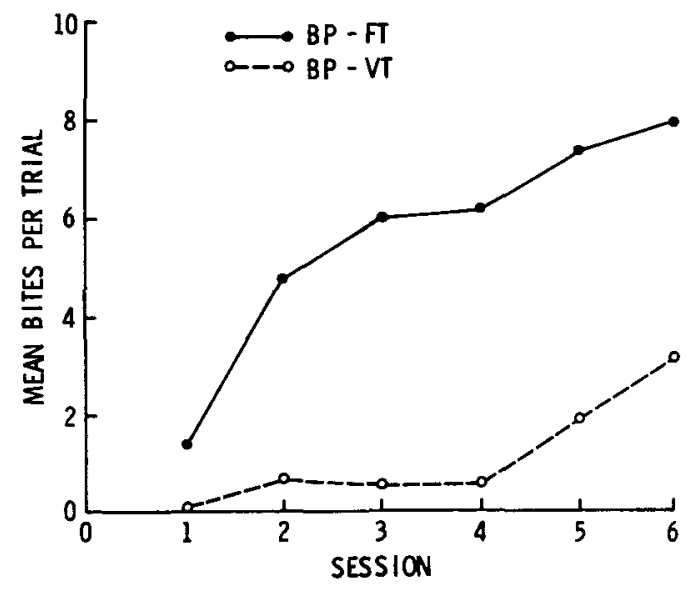

Figure 6. Average postshock bites per trial over Sessions 1-6. Data from test trials is not included.

Another estimate of the magnitude of the difference between Group BP-FT and Group BP-VT can be made in terms of the number of subjects exhibiting unconditioned and conditioned biting. In Group BP-FT 6/6 subjects displayed postshock biting, while $4 / 6$ bit the tube on shock-free trials. However, in Group BP.VT, only $4 / 6$ subjects exhibited postshock biting, while only 26 bites in total were made by 3 subjects over all test trials.

The proportion of the ITI which subjects spend in the forward half of the chamber did not differ between the groups $(F=1.0, \mathrm{df}=1 / 10, \mathrm{p}<.05)$. Figure 7 displays the mean proportion of ITI and CS periods spent in the forward half of the chamber for each group. Again, the proportion of the ITI spent forward was substantially greater than $50 \%$. During the CS periods Group BP-FT spent significantly more time forward than did Group BP-VT $(F=6.00, \mathrm{df}=1 / 10, \mathrm{p}<.05)$. Note that despite the tendency for Group BP.VT to move away from the tube, the proportion of the CS period spent near the tube remained near $70 \%$.

The results of the second experiment clearly demon-

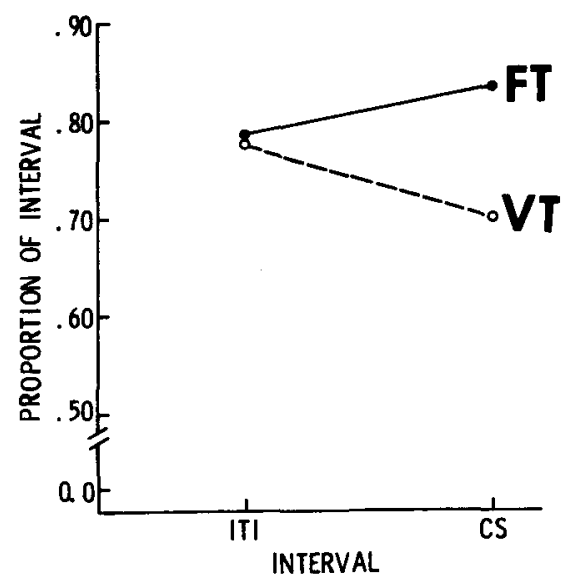

Figure 7. Mean proportions of ITI and CS intervals spent i the front half of the chamber. All trials over Sessions 1.7 are included. strated that the rapid conditioning of pain-elicited biting obtained with a backward pairing procedure depends upon the presentation of conditioning trials on a fixed time schedule. When trials were presented on the variable time schedule, very low levels of conditioned biting were observed.

Presentations of the shock-tube pairings on the variable time schedule not only eliminated stimulus control of biting, but effectively reduced the amount of postshock biting as well. Hence, we can conclude that the conditioned biting observed in the first experiment, and in Group BP-FT of the present experiment, are not examples of backward excitatory conditioning or escape conditioning contingencies. The data are, however, compatible with the "irnplicit" avoidance contingency analysis offered by Dunham.

\section{GENERAL DISCUSSION}

It has been suggested that the response elicited by aversive stimulation enjoys, in certain contexts, the property of predicting a shock-free interval longer than any other response in the organism's repertoire. The data from our initial experimentation with the phenomenon of pain-elicited aggressive behavior suggests that the organism's aggressive behavior is affected by the presence of this "implicit" avoidance contingency.

The data in both Experiments I and II suggest that the facilitation of aggressive behavior produced by periodic, unsignaled shock (Hutchinson, Renfrew, \& Young, 1971) is a product of the "implicit" avoidance contingency and not the excitatory classical conditioning mechanism initially suggested. In Experiment II, for example, the biting response as a CR and as a UCR increased over sessions, and was conditioned only if the fixed-time intertrial interval was employed.

The role played by the "implicit" avoidance contingency in the classical conditioning of aggressive behavior is less clear at present. We were not able to obtain strong evidence for conditioning in the forward pairing group of Experiment I, even when the fixed-time intertrial interval did define such an avoidance contingency. A number of factors were suggested to interfere with such conditioning and further research will be necessary to discover why our efforts, and a number of others, have failed to produce impressive conditioning of pain-elicited aggression. It was suggested, in particular, that incompatible, fear-motivated responses might interfere with the development of biting. Wasserman, Franklin, and Hearst (1974) have suggested in another context that animals will approach safe, positive conditioned stimuli and withdraw from aversive conditioned stimuli. Indeed, such observations raise the question of whether or not any "safety" stimulus presented to the monkeys in our procedure will acquire the property of eliciting biting behavior. We are examining this possibility at the present time, using variations on an explicitly unpaired procedure.

In summary, the present research provides evidence that the "implicit" avoidance contingency which is built 
into a number of procedures for presenting aversive stimulation to an organism does produce changes in the organism's behavior not observed in the absence of that contingency. A number of questions remain to be answered concerning the operation of this contingency in the context of other signaled and unsignaled aversive stimulation procedures.

\section{REFERENCES}

Creex, T. L., Hitzing, E. W., \& Schaeffer, R. W. Classical conditioning of reflexive fighting. Psychonomic Science, 1966 , 4, 89-90.

Dunham, P. J. Punishment: Method and theory. Psychological Review, $1971,78,58-70$.

Heth, C. D., \& Rescorla, R, A. Simultaneous and backward fear conditioning in the rat. Journal of Comparative and Physiological Psychology, 1973, 42, 434-443.

Hutchinson, $R$. $R$. The environmental causes of aggression. In J. Cole and D. Jensen (Eds.), Nebraska Symposium on Motivation, 1972. Lincoln: University of Nebraska Press, 1973.

Hutchinson, R. R., Azrin, N. H., \& Hake, D. F. An automatic method for the study of aggression in squirrel monkeys. Journal of the Experimental Analysis of Behavior, 1966,9 233-237.

Hutchinson, R. R., \& Emley, G. S. Schedule-independent factors contributing to schedule-induced phenomena. In $R$. $M$. Gilbert and J. D. Keehn (Eds.), Schedule effects: Drugs, drinking, and aggression. Toronto: University of Toronto Press, 1972.

Hutchinson, R. R., Renfrew, J. W., \& Young, G. A. Effects of long-term shock and associated stimuli on aggressive and manual responses. Journal of the Experimental Analysis of Behavior, 1971, 15, 141-160.
Lyon, D. O., \& Ozolins, D. Pavlovian conditioning of shock elicited aggression: A discrimination procedure. Journal of the Experimental Analysis of Behavior, 1970,13, 325-331.

Moore, B. R. The role of directed Pavlovian reactions in simple instrumental learning in the pigeon. In R. A. Hinde and J. S. Hinde (Eds.), Constraints on learning; limitations and predis. positions. London: Academic Press, 1973.

Moscovitch, A. \& LoLordo, V M. Role of safety in the Pavlovin a backward fear conditioning procedure. Journal of Comparative and Physiological Psychology, 1968, 66, 673-678.

O'Kelly, L. E., \& Steckle, L. C. A note on long-enduring emotional responses in the rat. Journal of Psychology, 1939, 8 , 125-131.

Rachlin, $H$. Autoshaping of key-pecking in pigeons with negative reinforcement. Journal of the Experimental Analysis of Behavior, $1969,12,521-531$

Rescorla, R. A. Pavlovian conditioning and its proper control procedures. Psychological Review, 1967, 74, 71-80.

Rescorla, R. A., \& Solomon, R. L. Two-process learning theory : Relationships between Pavlovian conditioning and instrumental learning. Psychological Review, 1967, 74, 151-182.

Rodger, R. S. Type I errors and their decision basis. British Journal of Mathematical and Statistical Psychology, 1967, 20, 51-62 (a).

Rodger, R. S. Type II errors and their decision basis. British Journal of Mathematical and Statistical Psychology, 1967, 20, 187-204 (b).

Siegel, S. \& Domjan, M. Backward conditioning as an inhibitory procedure. Learning and Motivation, 1971, 2, 1-11.

Vernon, W., \& Ulrich, R. E. Classical conditioning of painelicited aggression. Science, $1966,152,668-669$

Wasserman, E. A. Franklin, S. R \& \& Hearst, E. Pavlovian appetitive contingencies and approach versus withdrawal to conditioned stimuli in pigeons. Journal of Comparative and Physiological Psychology, 1974, 86, 616-627.

(Received for publication June 6, 1975 .

Revision received August 25, 1975.) 